\title{
Cytoreductive Surgery and Hyperthermic Intraperitoneal Chemotherapy Improves Survival of Patients with Peritoneal Carcinomatosis from Gastric Cancer: Trial Results of a Phase 2, Randomized, Clinical Trial
}

\author{
Peng Wang, MD and Zhi-xiang Zhou, MD
}

Department of Colorectal Surgery, National Cancer Center/Cancer Hospital, Chinese Academy of Medical Sciences and Peking Union Medical College, Beijing, China

\section{DEAR EDITOR:}

With great interest, we have read the phase 2, randomized, clinical trial published in the Annals of Surgical Oncology (2011;18(6):1575-81) by Yang et al. Great efforts have been made to prove that synchronous gastric peritoneal carcinomatosis (PC), cytoreductive surgery (CRS) + hyperthermic intraperitoneal chemotherapy (HIPEC) with mitomycin C $30 \mathrm{mg}$, and cisplatin $120 \mathrm{mg}$ may improve survival with acceptable morbidity.

It is interesting that cases in the CRS + HIPEC group have a 4.5-month longer median survival than that in CRS group $(P=0.046)$ with a median follow-up of 32 months.
As the desirable result, this study may affect the decision of whether HIPEC should be used for cases with gastric PC or not. They did great work.

However, in our opinion, improper annotations at the bottom of the Fig. 1a and b were published. The appellations of the two groups are inconsistent with the explanations in the text. It is important for a correct figure to convey a desirable result, or a misunderstanding could be made.

DISCLOSURE There are no conflicts of interest.

(C) Society of Surgical Oncology 2017

First Received: 12 October 2017;

Published Online: 26 October 2017

Z. Zhou, MD

e-mail: zhouzx001@163.com 